\title{
SORTIR DU JOURNALISME. LES DIPLÔMÉS EN JOURNALISME ENTRE EMPLOIS INSTABLES ET CARRIÈRES DÉVIANTES
}

\author{
Valérie DevillarD $^{1}$, Guillaume Le SAULNIER ${ }^{2}$
}

\begin{abstract}
L'article se concentre sur les parcours des diplômés en journalisme conduits à quitter la profession à laquelle ils se destinaient. Il se fonde pour cela sur des entretiens auprès de trente-quatre diplômés, recrutés via LinkedIn au sein de quatre promotions (1997, 2007, 2012, 2017). Tant les conditions objectives d'engendrement des « sorties » du journalisme que les opérations subjectives qui les soutiennent y sont examinées. Les récits de parcours insistent sur la dégradation des conditions d'insertion et d'emploi dans les métiers du journalisme. Les diplômés cantonnés à des emplois instables construisent fréquemment leur carrière dans un va-et-vient entre des emplois journalistiques et non journalistiques, où s'élaborent des contre-modèles professionnels qui préfigurent leur défection. Les carrières « divergentes " peuvent ainsi être pensées comme des carrières "déviantes", au sens où elles engagent une série d'écarts ou de transgressions vis-à-vis des standards par lesquels se définissent les « journalistes professionnels ».
\end{abstract}

«Le journalisme mène à tout, à condition d'en sortir ». Cette observation, attribuée à l'écrivain, critique et journaliste Jules Janin (18041874), résume la difficulté de faire carrière, mais aussi la dureté et l'ins-

1 Valérie Devillard, Carism, Université Panthéon-Assas Paris 2.

2 Guillaume Le Saulnier, Cérep, Université de Reims Champagne-Ardenne.

Recherches en communication, $\mathrm{n}^{\circ} 43$ - Article publié le 13/08/2020 
tabilité des conditions d'emploi au temps de l'industrialisation de la presse. Si elle s'est muée en cliché, elle a conservé, plus d'un siècle après et nonobstant la professionnalisation des journalistes opérée dans l'intervalle, une partie de son actualité. En effet, le journalisme contemporain se confronte à une crise structurelle qui se matérialise dans la fragilité de l'économie de la presse, la dualisation de plus en plus accusée des marchés du travail, la précarisation croissante des journalistes, ou encore la dégradation de leurs conditions de travail. Cette crise se conjugue avec des processus d'individualisation des carrières (Bastin, 2015 ; Standaert, 2016b), au sein de trajectoires modulées par les difficultés d'insertion, l'extension des emplois instables, l'hétérogénéité des statuts, la discontinuité des parcours et l'accélération des sorties de la profession (Devillard \& Rieffel, 2001 ; Devillard, 2002 ; Frisque \& Saitta, 2011 ; Scam, 2013 ; Leteinturier, 2016). Gilles Bastin constate ainsi « l'étranglement des carrières produit par la diminution de l'âge de sortie des journalistes des mondes de l'information et la multiplication des parcours instables dans ces mondes » (Bastin, 2015, p. 147).

Dès lors, que signifie aujourd'hui « sortir » du journalisme ? D'où viennent et que deviennent les « sortants », conduits à quitter la profession à laquelle ils se destinaient? Quelles sont les conditions objectives qui engendrent ces défections ? Quelles sont les opérations subjectives qui les soutiennent? Suivant le paradigme « gravitationnel » des mondes de l'information (Bastin \& Machut, 2016), quelles sont les expériences et les épreuves qui jalonnent et façonnent la « divergence» des trajectoires professionnelles?

Paradoxalement, au regard de la dégradation de l'économie de la presse, on constate un déséquilibre dans la recherche en sciences sociales autour des carrières au sein des mondes de l'information. On recense de nombreux travaux sur la socialisation professionnelle des journalistes, à commencer par les instances de formation et les conditions d'insertion (Marchetti \& Ruellan, 2001 ; Lafarge \& Marchetti, 2011 ; Standaert, 2016a). Les enquêtes se concentrent également sur la condition des journalistes précaires, l'extension des emplois instables ou flexibles, et les carrières qui se construisent dans les seuils ou les marges du groupe professionnel (Accardo, 1998 ; Frisque \& Saitta, 2011 ; Standaert, 2016b). Mais les parcours et les devenirs des « sortants » des métiers du journalisme restent peu documentés, hormis quelques travaux récents (Frisque, 2014 ; Leteinturier, 2014 ; Bastin, 2015 ; Bouron, Devillard, Le Saulnier \& Leteinturier, 2017 ; Lafarge, 2019). 
Nous nous concentrerons ici sur les sorties ${ }^{3}$ du journalisme, c'està-dire autant sur les engagements professionnels que sur les désengagements, les défections, les déplacements, les bifurcations, les reconversions, et la série d'ajustements individuels qu'ils charrient. Nous mobiliserons pour cela le concept de « carrière » au sens que lui donne la sociologie interactionniste des professions (Hughes, 1996, pp. 165185 ; Darmon, 2008). Dans cette acception, la carrière des individus se construit de façon processuelle et négociée, dans une dialectique perpétuelle entre les dimensions objectives structurantes des trajectoires et des activités (l'état des marchés du travail, les logiques sectorielles et organisationnelles, les relations professionnelles, les conditions d'emploi et de travail, les positions et les dispositions sociales) et les dimensions subjectives des biographies et des expériences (les aspirations, les frustrations, les définitions des situations et des identités au travail).

Sous ce prisme, nous examinerons les origines et les devenirs des carrières bloquées, lesquelles peuvent être pensées et vécues comme des carrières " déviantes " à l'aune des positions et des attributs qui incarnent l'excellence professionnelle journalistique. En effet, les parcours des « sortants » se construisent fréquemment dans un continuum de situations professionnelles, et, plus spécifiquement, dans un va-etvient entre des emplois journalistiques et non journalistiques, et se donnent ainsi comme l'envers, sinon la transgression, des standards qui soutiennent la professionnalisation des métiers du journalisme. A commencer par le mélange des genres entre le journalisme et la communication, lequel est aussi fermement réprouvé par le groupe professionnel qu'il est fréquemment assumé par celles et ceux qui en occupent les marges (Frisque, 2014).

\section{Des formulaires LinkedIn aux récits de parcours}

La recension des « sortants » des métiers du journalisme a été réalisée via LinkedIn, réseau social à vocation professionnelle ${ }^{4}$. En effet, de

3 La notion de « sortie » désigne ici le fait de quitter (de façon précoce ou tardive, absolue ou relative) les métiers du journalisme pour se diriger vers des emplois non journalistiques. Elle diffère ainsi des études autour des « sortants » des fichiers de la Commission de la carte d'identité des journalistes professionnels, c'est-à-dire des titulaires de la carte de presse dont la demande n'a pas été renouvelée ou a été refusée par la Commission.

4 Nous tenons à remercier vivement les étudiants des promotions 2015-2016 et 20192020 du master 2 « Médias, publics et cultures numériques » de l'Institut Français de 
nombreux journalistes (anciens ou actuels) y disposent d'un « profil » à des fins de visibilité. Les profils, auto-déclarés dans un formulaire commun à tous les usagers, sont à l'image d'un curriculum vitae : y figurent leur nom, leur fonction et leur organisation actuelles, leur lieu de résidence, les séquences estimées les plus significatives de leur carrière, et, pour chacune, les dates de début et de fin, ainsi que les formations universitaires suivies, les établissements et les années de délivrance des diplômes. Un espace rédactionnel ouvert permet une description plus personnelle ou stylisée. Les profils peuvent aussi donner à voir les réalisations et les centres d'intérêt des usagers, les groupes auxquels ils sont affiliés, ou encore les compétences « recommandées » par leurs interconnaissances en ligne. En vertu du caractère déclaratif et uniforme des informations publiées sur le réseau social, celui-ci fournit une base de données propice à une analyse séquentielle des carrières (Bastin, 2016). Parmi ses avantages, il permet de recenser les « sortants » des métiers du journalisme et de documenter leurs parcours, au contraire des fichiers administratifs de la Commission de la carte d'identité des journalistes professionnels (CCIJP), pour laquelle les devenirs des « sortants » de la carte de presse restent une inconnue.

En l'occurrence, la recension effectuée sur LinkedIn s'est fondée sur trois critères : la détention d'un diplôme de formation en journalisme, qu'elle soit « reconnue » ou non par la profession ; l'année de délivrance du diplôme (1997, 2007, 2012, 2017), l'enquête par entretiens étant associée à une étude quantitative longitudinale auprès de trois cohortes de nouveaux titulaires de la carte de presse (1998, 2008, 2013) (Bouron, Devillard, Le Saulnier, Leteinturier, 2017) ; enfin, le caractère non journalistique du dernier emploi déclaré sur le réseau social. Des biais sont à signaler dans les résultats de la recension : le critère du diplôme en journalisme écarte les individus ayant exercé dans la presse sans avoir suivi une formation spécialisée ; de surcroît, les diplômés d'écoles reconnues par la profession sont plus nombreux que les lauréats de formations non reconnues, car des métadonnées « formation » sont généralement disponibles pour les premières, ce qui accroît sensiblement le nombre et la pertinence des résultats. Lors de la phase de recrutement des enquêtés, nous nous sommes efforcés de corriger partiellement ce dernier biais.

Presse (Université Panthéon-Assas Paris 2) pour leur précieuse contribution à cette enquête. 
L'échantillon se compose ainsi de trente-quatre individus diplômés en journalisme, dont ce métier a constitué une séquence plus ou moins longue dans leur carrière, ou, pour cinq d'entre eux, est devenu une activité reléguée au second plan. Les promotions y sont représentées de façon à peu près égale, à l'exception de la dernière (2017) qui reste très minoritaire. La grande majorité des individus sont dotés d'un diplôme de master et, pour les autres, d'une licence. Vingt-deux diplômés émanent d'écoles reconnues par la Commission paritaire nationale de l'emploi des journalistes (CPNEJ) ; les douze restants sont issus de formations publiques ou privées spécialisées en journalisme mais non labellisées.

L'échantillon présente un déséquilibre important dans la distribution entre les femmes et les hommes (respectivement vingt-cinq et neuf). Il réfracte ainsi, à l'instar de précédentes enquêtes, la structuration fortement genrée des carrières, qui discrimine les femmes avec des parcours plus chaotiques, des statuts plus précaires, une ascension freinée par le plafond de verre, certes fissuré dans les années 2000 (Cardon $\&$ Roharik, 2016) mais encore bien présent. Les femmes journalistes connaissent dès lors des périodes de chômage plus fréquentes et des sorties de la profession significativement plus nombreuses.

Majoritairement issues des classes moyennes supérieures, les femmes disposent de ressources scolaires qui caractérisent (en volume et en structure) les parcours d'excellence universitaire : classes préparatoires littéraires, diplômes de Sciences Po ou des instituts d'études politiques (IEP de Lille, Lyon ou Rennes), ou encore doubles cursus. Seize d'entre elles sont diplômées d'écoles reconnues, dont certaines parmi les plus prisées sur le marché des formations en journalisme. Les origines sociales des hommes sont plus contrastées, et six d'entre eux sont parvenus à intégrer une formation en journalisme labellisée. Les secteurs de reconversion se distribuent de façon à peu près équivalente entre les femmes et les hommes : elles s'opèrent pour vingt-deux individus dans des métiers connexes à ceux du journalisme, à commencer par la communication d'entreprise, le « journalisme » institutionnel ou territorial, mais aussi le marketing digital, la création de contenus numériques, l'édition ou l'audiovisuel. Les douze diplômés restants se sont dirigés vers des métiers sans lien avec le journalisme, dans les secteurs de l'immobilier, de la banque, de la vente, ou encore du tourisme. Pour l'essentiel, les conditions d'emploi au terme de la reconversion suggèrent une recherche de stabilité : on dénombre jusqu'à quinze salariés en contrat à durée indéterminée (CDI) - parmi lesquels deux fonction- 
naires territoriaux - dont une grande majorité sont des femmes. Elles reflètent également l'extension des statuts flexibles, où les femmes sont proportionnellement plus nombreuses que les hommes, avec sept diplômés exerçant désormais comme freelance, auto-entrepreneur ou micro-entrepreneur.

Les entretiens réalisés au sein de cet échantillon sont des récits de parcours ayant vocation à documenter la carrière des individus, les séquences qui la composent, les transitions et les éventuelles continuités de l'une à l'autre, les mobilités professionnelles et les conditions dans lesquelles elles s'opèrent, ainsi que le sens subjectif donné aux engagements et aux désengagements successifs, à commencer par la sortie des métiers du journalisme. Si elle permet de reconstituer finement les carrières individuelles, et de sonder leur dimension expérientielle et les justifications qui les soutiennent, cette méthode d'enquête engendre ses propres biais. Le premier d'entre eux consiste à interviewer des professionnels de l'enquête, lesquels se montrent fréquemment à l'aise et coopératifs dans cette situation d'interlocution spécifique, mais aussi particulièrement aptes à contrôler leur discours, voire tentés de diriger l'entretien. Un autre biais réside dans les difficultés éprouvées par certains enquêtés pour narrer les transitions d'une séquence à l'autre, les circonstances précises, les anticipations, les opérations et les éventuels aléas qui les font advenir. La troisième limite se loge dans la forte récurrence de deux assertions relatives aux chances d'insertion dans les métiers du journalisme : à savoir le passage, souhaitable sinon nécessaire, par une formation reconnue, et l'entremise décisive du « réseau », des « contacts », du « carnet d'adresses », pourvoyeurs d'opportunités professionnelles. Aussi fondées soient-elles, ces assertions muées en évidences suggèrent l'existence d'une doxa parmi les candidats aux métiers du journalisme, qui structure puissamment la mise en récit des carrières et fournit un schème préétabli pour expliquer les insertions contrariées. Les enquêtés sont ainsi tentés de mobiliser ces généralités pour narrer leur trajectoire dans et hors les mondes de l'information, alors qu'elles ne peuvent suffire à comprendre les carrières individuelles dans toute leur complexité et leur singularité. 


\section{Une dégradation des conditions d'insertion et d'emploi}

\subsection{Les barrières à l'entrée de la profession}

Les barrières à l'entrée dans le journalisme sont toujours plus difficiles à franchir pour les diplômés des promotions de 2007, 2012 et 2017, au regard de ceux de la génération de 1997. A l'instar de l'ensemble des trajectoires des diplômés de l'enseignement supérieur, les temps d'insertion s'allongent, avec des modalités de plus en plus complexes et, en corollaire, des cheminements davantage chaotiques (Lefresne, 2003). Les travaux sur les conditions d'entrée des jeunes journalistes (Marchetti \& Ruellan, 2001 ; Devillard \& Rieffel, 2001 ; Devillard, 2002 ; Leteinturier \& Frisque, 2015) ont montré combien les aléas que subissent certains parcours s'inscrivent dans les évolutions structurelles de l'emploi des jeunes et de leur mode de recrutement. Les années 1990 ont été marquées par un cycle de récession économique culminant entre 1991 et 1992, qui a eu des conséquences importantes sur les effectifs des journalistes. Ainsi, parmi les nouveaux titulaires de la carte de presse en 1990, un tiers de la cohorte avait disparu des fichiers de la CCIJP neuf ans après leur première carte de presse. Cette dynamique centrifuge se poursuit dans les années 2000. Les promotions de 2007 et 2012 ont été ainsi confrontées, dans leurs premières années de vie active, à la crise financière mondiale survenue en 2008 et à celle des dettes souveraines de 2012. Par exemple, Adrien, diplômé en 2007 du master journalisme de l'IEP de Rennes, devenu rédacteur en chef en CDI dans une collectivité territoriale qui édite ses propres supports, se réfère explicitement à la contraction de l'emploi consécutive à la récession due à la crise économique de 2008 :

«J'ai de la chance, je suis rentré dans le secteur du travail juste avant 2008, et effectivement dans mon entreprise où on m'avait embauché et où on avait embauché pas mal de journalistes, il y a eu des licenciements économiques peut-être un ou deux ans après, quoi. Du coup, il [le marché du travail] s'est endurci, mais il était déjà sacrement amoché » (Adrien, trente-sept ans, rédacteur en chef dans une collectivité territoriale, formation non reconnue, promotion 2007). 
La reprise économique trop faible n'a jamais permis un rattrapage des niveaux d'emploi d'avant 2008. Cette détérioration durable du marché de l'emploi forme la toile de fond où s'inscrit l'ensemble des parcours des « sortants » qui, pour la majorité d'entre eux, n'ont connu aucun aboutissement à leur phase d'insertion, ayant été reconduits de façon répétée dans des emplois instables. Ceux-ci sont devenus non plus tant l'emblème d'un statut précaire mais transitoire - le système des « pools » de contrats à durée déterminée (CDD), présentés comme des sas d'entrée pour l'accès à un emploi stable - que l'un des modes ordinaires de gestion de l'emploi des journalistes.

Ce contexte économique dégradé a fortement pesé sur les possibilités et les rythmes d'accès à l'emploi parmi les journalistes débutants : ceux-ci ont été davantage exposés à des périodes durables d'inactivité, de chômage ou de reprise d'études. Cela a également affecté les chances de progression de carrière. Parmi les diplômés en journalisme cumulant les ressources qui prédisposent à l'accès à des positions privilégiées dans les mondes de l'information - notamment le passage par une école reconnue, véritable sésame facilitateur d'insertion - nombreux sont ceux qui ont jeté l'éponge après quelques années d'exercice, malgré un parcours continu et sans accidents dans les métiers de la presse.

Cependant, la majorité des « sortants » interviewés ont été préalablement fragilisés par des emplois journalistiques exercés soit dans des conditions dégradées dans des secteurs structurés par le recours systématique et institutionnalisé à des statuts précaires (à l'instar de l'usage pour les salariés en CDD de la période de « carence » empêchant l'embauche en CDI) ; soit dans des secteurs de presse toujours plus affaiblis par la production d'une information quotidienne " gratuite », fournie par des professionnels du marketing, des producteurs de contenus web, sinon par des amateurs ; soit, encore, dans des emplois toujours plus proches des secteurs dans lesquels leur reconversion s'est ensuite opérée (par exemple, de la presse locale vers la communication territoriale, ou de certains secteurs de la presse technique et professionnelle vers le « journalisme» institutionnel ou d'entreprise).

\subsection{L'institutionnalisation de la précarité et la dualisation du marché de l'emploi}

Cette dégradation durable des possibilités et des conditions d'insertion a changé structurellement le marché de l'emploi journalistique. L'hémorragie des effectifs qui en a résulté pour partie, observée à 
plusieurs reprises dans les fichiers de la CCIJP (Devillard \& Rieffel, 2001 ; Devillard, 2002 ; Leinturier \& Frisque, 2015 ; Bouron, Devillard, Le Saulnier, Leteinturier, 2017), a trouvé une réponse univoque dans la précarisation des journalistes. Ceux-ci subissent un processus plus vaste qui frappe de plein fouet l'ensemble des professions intellectuelles.

Dans un premier temps, cette conjoncture détériorée a exercé des effets pérennes, tout particulièrement sur les débuts de l'emploi, avec un allongement de la durée de la phase d'insertion et, en corollaire, un accroissement des étapes professionnelles transitoires. Les plus touchés - avec une surreprésentation structurelle des femmes, lesquelles sont plus souvent une variable d'ajustement en cas de crise économique ont été les candidats à la profession dotés de ressources scolaires moindres et faiblement spécialisées en journalisme, et n'ayant pas ou peu thésaurisé de réseaux professionnels. A l'opposé, les diplômés des formations reconnues ont été affectés dans une bien moindre mesure dans leurs possibilités d'accès à l'emploi journalistique et ont occupé rapidement les postes stables (c'est-à-dire en CDI) situés en haut de la hiérarchie professionnelle (dans la presse quotidienne nationale, l'audiovisuel ou les agences de presse). D'après les précédentes enquêtes longitudinales, les diplômés d'écoles labellisées ou de formations spécialisées connaissent a priori des trajectoires davantage protégées, et ont plus de chances de traverser des parcours d'insertion moins longs et sinueux que l'ensemble des journalistes.

Il en a résulté une dualisation progressivement plus marquée de l'emploi journalistique dans les années 2010. La « saignée » des effectifs a renforcé les inégalités de parcours. Dans un second temps, le rôle protecteur des diplômes des écoles reconnues a ainsi été moindre, notamment pour la génération peu préparée au bouleversement du numérique dans l'emploi journalistique. Là encore, bien que les femmes soient souvent davantage diplômées que les hommes (certaines ayant une double diplomation en IEP et dans une formation reconnue de niveau master), c'est l'emploi féminin qui a été plus particulièrement affecté ; leur carrière étant déjà freinée par le plafond de verre qui ralentit leur accès voire empêche leur ascension vers les rares postes de direction.

La contraction de l'offre d'emploi est visible également dans les rémunérations modestes liées à la précarité des statuts où sont cantonnés des diplômés pourtant fortement dotés en ressources scolaires. Béatrice, diplômée en 2012 d'une licence professionnelle non reconnue, 
demandeuse d'emploi au moment de l'enquête, commente les vicissitudes de son parcours et, au rebours des récits dominants, souligne les avantages procurés par les formations non labellisées dans les chances d'insertion :

«Faire cinq ans d'études pour être journaliste et voir comment tu galères financièrement et même pour te faire une place dans le métier, ça ne vaut pas le coup. » Elle ajoute, comparant la situation des diplômés des formations reconnues avec celle des autres diplômés : «Tu leur coûtes moins cher qu'un journaliste qui a cinq ans d'expérience. Car tu es moins payée, ce qui les intéresse encore plus. Je crois d'ailleurs que ce sont les journalistes qui n'ont pas fait d'écoles reconnues car ils sont payés encore moins que ceux qui sortent d'écoles reconnues. Et puis eux ils restent deux ans stagiaires, alors que nous on ne reste qu'un an stagiaire » (Béatrice, vingt-neuf ans, consultante et formatrice en communication au chômage, formation non reconnue, promotion 2012).

Au-delà de la conjoncture défavorable qui freine leur entrée dans la vie active, cette génération de diplômés a dû composer avec les transformations des modes d'embauche, qui privilégient toujours plus les différentes formes de contrats à durée limitée. Cette évolution a modulé pleinement les conditions de recrutement, et nous avons vu combien elle est désignée comme l'indicateur phare de la précarité au sein de l'échantillon de " sortants » des métiers du journalisme. Ainsi, si l'on dessinait leur portrait-robot, il aurait les contours d'une femme (certes plus nombreuses dans les promotions des nouveaux « entrants ", mais aussi et surtout dans les sorties précoces), statutairement pigiste (dont le nombre a presque été multiplié par trois en l'espace de trente ans) ou salariée en CDD, et exerçant dans des secteurs médiatiques où l'offre d'emploi se contracte structurellement (à savoir la presse spécialisée grand public, la presse quotidienne régionale, les radios ou télévisons publiques).

\section{Faire défection : les récits de sortie du journalisme}

Parmi les raisons avancées par les " sortants » des métiers journalisme pour expliquer leur reconversion, la dégradation des condi- 
tions d'emploi et de revenu est quasi systématiquement évoquée. Cela concerne aussi bien les départs précoces (quelques mois après la délivrance du diplôme), au nombre de dix dans l'échantillon, que les sorties progressives ou tardives (après plus de sept années d'exercice dans la profession).

\section{1. "La désillusion"}

Certains « sortants » précoces ont connu pour unique carrière en journalisme celle consacrée à leurs années d'études dans l'une des formations spécialisées. Leurs seules expériences sont les stages, les contrats d'alternance ou de professionnalisation, ou, parfois, les CDD effectués voire accumulés au cours de leur scolarité. Ainsi, rien ne prédestinait au journalisme Léo, diplômé en 2012 d'une formation non reconnue, si ce n'est sa familiarité avec une profession exercée par sa mère. Bien au contraire : «Ma mère est journaliste, raconte-t-il, et $d u$ coup je ne voulais absolument pas faire ça quand j'étais petit. [...] J'ai toujours bien aimé écrire mais je ne me suis jamais vu comme quelqu'un qui allait écrire des articles, quoi, vraiment pas spécialement. » Les études en journalisme ont pourtant attiré ce sportif de haut niveau (dont le père est professeur d'éducation physique et sportive) après son bac littéraire, car l'une des formations proposait un cursus de journalisme en sport-études. Léo s'oriente ensuite vers le master en urbanisme de Sciences Po, puis le master de paysagiste-concepteur de 1'Ecole Nationale Supérieure du Paysage de Versailles. Les stages effectués lors de sa formation en journalisme ont agi sur lui comme un repoussoir et mis un terme à toute velléité de poursuivre dans cette voie. Il décrit ainsi l'une des expériences qui l'ont dissuadé de faire du journalisme :

«Et surtout une expérience, dans une espèce de magazine en ligne, sorte de GQ à la française, ils faisaient des pages cachées, en fait des espèces de pages [...] avec 120000 conditionnels pour parler du futur transfert de tel Brésilien au PSG [Paris Saint-Germain] et pour alimenter des rumeurs à fond. Voilà, et ça nous permettait de faire tant de milliers de vues, pour par la suite être mieux vendus aux annonceurs, et ça voilà c'était pas appréciable. Puis faut faire un article en cinq minutes avec 12000 mots-clés sans obligation de faire quelque chose de propre et bien 
écrit, quoi » (Léo, vingt-neuf ans, architecte-paysagiste en CDD, formation non reconnue, promotion 2012).

D'autres « sortants » précoces des métiers du journalisme, dépendants des réseaux tissés lors de leur formation pour surmonter la période d'instabilité qui s'ensuit, n'ont pas capitalisé les compétences relationnelles données comme consubstantielles à l'exercice de la profession. Faute d'avoir respecté cet impératif de tout « bon » journaliste - enseigné dans les formations spécialisées - et d'avoir su ou pu développer l'ethos professionnel attendu, ils sont vite contraints de renoncer à leurs ambitions et de se diriger vers d'autres secteurs d'activité.

Ces débuts de carrière sont vécus comme autant d'expériences limites dans le journalisme, qui expliquent les défections des " sortants » de la première heure via une poursuite d'études (dans le marketing digital, le paysagisme, les gender studies), dans des métiers connexes à ceux de la presse (en premier lieu la communication), ou encore dans l'entreprenariat (dans le conseil, la formation, la vente).

\section{2. "Tenir bon "}

Le passage " obligé » par les stages, les piges et autres statuts précaires - encore donnés comme des sas d'entrée nécessaires mais, depuis longtemps, bien souvent insuffisants - pour accéder à un emploi stable dans la presse rebute plus d'un candidat à la profession. Pareille mise à l'épreuve inaugure et préfigure un "métier de sacrifices 》, décrit en ces termes ou selon des figures analogues par plusieurs enquêtés. En témoigne Sylvain, diplômé en 1997 de l'Ecole de journalisme et de communication d'Aix-Marseille (EJCAM), désormais auto-entrepreneur dans la vente, qui a délaissé sa première vocation pour se lancer dans « une vie aventureuse » de responsable de téléphonie mobile sur le continent africain :

«J'avais la possibilité de poursuivre dans le journalisme [comme salarié en CDI pour Nice-Matin] mais je ne devais pas suffisamment aimer le journalisme à la rigueur parce que je ne voulais pas en baver, quoi. [...] Je ne voulais pas faire des chroniques de chiens écrasés à Nice » (Sylvain, quarante-neuf ans, auto-entrepreneur dans la vente, formation reconnue, promotion 1997). 
La recherche de la stabilité économique et sociale que procure le salariat en CDI représente l'un des arguments les plus récurrents, dans ces récits de parcours, pour justifier a posteriori le renoncement à faire carrière dans le journalisme. En atteste la nette prévalence des salariés en CDI (au nombre de quinze) parmi les divers statuts exercés par les enquêtés au terme de leur reconversion, auxquels s'ajoutent les trois postes de contractuel de la fonction publique d'Etat ou territoriale. Cette quête de stabilité imprègne aussi le récit des journalistes installés dans des emplois instables, sous des statuts des plus précaires, tels les « bénéficiaires » de CDD à répétition, les pigistes, les indépendants, les auto-entrepreneurs, voire les intermittents du spectacle.

L'obtention d'un CDI aussitôt après la formation représente l'exception, tant ce statut est devenu "une denrée extrêmement rare ", et ce, même après deux ans d'expérience dans la profession. Marine, community manager âgée de vingt-huit ans, décrit ainsi son incrédulité lorsque, à l'issue d'un stage de fin d'études dans le cadre d'une licence professionnelle obtenue en 2012, elle est embauchée en CDI au Mans par la rédaction d'un pure player spécialisé dans les voyages, avant d'être licenciée économique au bout de neuf mois :

"Oui, j'étais en CDI, alors euh le truc, le truc unique, quoi! Enfin, t'es journaliste, t'es jamais embauchée en CDI! J'aurais dû m'en méfier, quoi... C'était un contrat qui,je pense, au niveau fiscalité, toutça, arrangeait pas mal plutôt qu'un CDD à renouveler de temps en temps, etc. Et puis, on n'était vraiment pas beaucoup dans l'entreprise. On n'était que deux journalistes, journalistes carte de presse, et après c'était des pigistes qui travaillaient pour différentes cibles " (Marine, vingt-huit ans, community manager en agence, formation non reconnue, promotion 2012).

Le rejet de cette insécurité professionnelle parmi les enquêtés peut expliquer amplement leur décrochage du journalisme. Leurs propos font chorus pour décrire les peines, sinon les souffrances, engendrées par l'exercice du métier dans des conditions maintes fois décrites comme difficiles. Ils expriment ainsi «l'usure», tant physique que psychologique, qu'ils ont pu ressentir ou ressentent encore en relatant des situations de quasi burn out, tant les cadences de travail et la pression de la concurrence ont failli les épuiser. En témoigne Anémone, diplômée 
en 2012 de l'Ecole publique de journalisme de Tours (EPJT), devenue directrice de communication dans une communauté de communes du Sud de la France, après avoir été licenciée en 2016 de son poste de journaliste reporter, la veille de la signature d'un CDI promis depuis un an par ses employeurs. Cette sortie subie "dans le sang et la fureur" a succédé à maints CDD enchaînés entre 2012 et 2016 dans le même groupe de presse régionale. Elle expose ainsi son expérience au sein d'un «pool de $C D D$ » et la gestion des emplois pratiquée au sein du groupe de presse :

«Vous voyez j'ai travaillé de janvier 2015 à septembre 2015 au siège, et après j'avais enchaîné deux CDD. Après il y a ce qu'on appelle la carence, c'est-à-dire qu'on ne peut pas faire plus de trois CDD consécutifs, sinon on est obligés de nous embaucher en CDI, donc on nous met en carence pendant trois mois, et au bout de deux mois et demi on commence à rappeler en disant (imitant d'une voix enjouée) : "Coucou! Vous vous rappelez, bientôt la carence c'est terminé." [...] Donc on rappelle tout le temps, et faut que tu aies le pied dans la porte » (Anémone, trente-deux ans, direction de communication, contractuelle de la fonction publique, formation reconnue, promotion 2012).

Ce mode de recrutement, déjà installé à la fin des années 1990 dans la presse locale, forme le pendant de celui en vigueur dans les radios publiques locales, tout aussi contraignant (à savoir l'inscription au planning national du réseau). Corentin, directeur d'une agence de conseil en communication, âgé de quarante-six ans, diplômé de l'IEP de Lille et (en 1997) du Centre universitaire d'enseignement du journalisme de Strasbourg (CUEJ), cherche ainsi un emploi durant la phase de carence de son premier CDD dans la presse régionale, et intègre une agence de communication et relations presse. Il relègue définitivement au second plan le journalisme, pourtant désigné comme une « vocation », pour lui préférer la stabilité et les perspectives de carrière promises dans le secteur de la communication. Le journalisme devient un simple hobby pour ce fils de grand reporter, qui déclare avec une légère amertume : «On avait le journalisme dans le sang dans la famille. » 


\subsection{Se découvrir une "fibre entrepreneuriale "}

Beaucoup de « sortants » diplômés des formations les plus réputées estiment avoir écrit leurs meilleurs papiers au cours de leur scolarité, avant de revoir leurs exigences à la baisse dans leur pratique professionnelle, à cause de la «détérioration des conditions de travail et de l'environnement économique et politique ». Leur vocation pour le journalisme a cédé le pas face aux déceptions et aux difficultés rencontrées dans son exercice au quotidien, jusqu'à éprouver des sentiments d'amertume, de regret, de nostalgie, et enfin d'impuissance à pouvoir changer le cours de leur carrière et à revenir en arrière.

La recherche d'une autonomie professionnelle en vue d'échapper à ce genre d'aléas représente un motif récurrent dans les récits de reconversion via l'entrepreneuriat, qu'il s'agisse de créer et de diriger une société ou d'exercer sous le statut d'indépendant. L'entrepreneuriat étant parfois donné comme un moyen de renouer avec certaines facettes du métier de journaliste, notamment l'esprit d'initiative, le sens du contact, 1' « intelligence des gens et des situations ». C'est le cas de Christelle, cheffe d'une micro-entreprise de quarante-six ans, diplômée en 1997 de l'Ecole supérieure de journalisme (ESJ) de Lille : journaliste pour la télévision, elle débute parallèlement des activités de media training pour compléter ses revenus, avant de créer une micro-entreprise et de se lancer à temps plein dans le métier de coach en prise de parole. Elle insiste sur les bénéfices primaires et secondaires que l'entrepreneuriat lui procure :

«D'abord dans un premier temps, [je me suis lancée] pour des raisons purement économiques, c'est-à-dire que moi j'avais besoin de compléments de revenu en plus de mes piges, donc forcément, la façon la plus rapide de gagner un peu plus d'argent, notamment quand on est journaliste télé, c 'est à côté de faire du media training. Donc voilà, ça a été la première raison. La deuxième raison c'est que, je l'ignorais moi-même, mais au fond de moi sommeillait une cheffe d'entreprise. C'est-à-dire que j'avais très envie de prendre mon destin en main et de ne plus être dépendante des différentes demandes des rédactions que j'avais en face de moi. Quand on travaille en télévision il y a un côté très compliqué, très précaire, et on est complètement à la merci des uns et des autres. Il y avait donc cette fibre entre- 
preneuriale. Et puis pour une raison personnelle pour le coup, c'est que moi j'ai adoré mon métier de journaliste mais il m'a énormément fatiguée (en riant) » (Christelle, quarante-six ans, coach en prise de parole, micro-entrepreneure, formation reconnue, promotion 1997).

\section{4. "Un métier de sacrifices"}

Les récits de parcours décrivent comment, corvéables à merci, certains jeunes journalistes n'ont pas compté leurs heures pour produire toujours plus de contenus d'actualité dans des délais toujours moindres, afin de prouver leur engagement professionnel auprès de leurs employeurs. Cette " passion » pour le métier dévore la vie privée et peut, le cas échéant, faire obstacle à l'établissement d'une vie conjugale et parentale. Marine, sortante « précoce » de la profession, n'envisage pas de revenir aux métiers du journalisme, si ce n'est celui connexe de community manager au service d'une entreprise de presse, car il lui semble peu probable de pouvoir concilier journalisme et vie de famille :

«Le journalisme, en tout cas dans le domaine de la presse quotidienne régionale et de la presse hebdomadaire, tu fais des horaires de folie, quoi. Pour avoir vu au cours de mes stages des mères de famille ou même des journalistes célibataires, des fois je pense qu'ils étaient un peu au bout du rouleau, de courir à droite à gauche, terminer à minuit un conseil municipal où on a voté trois lois sur l'eau qui s'évacuait pas bien, voilà ! » (Marine, vingt-huit ans, community manager en agence, formation non reconnue, promotion 2012).

Certains récits de carrière font ainsi de la vie privée l'un des leviers explicatifs des bifurcations professionnelles : mobilité géographique du conjoint, opportunité d'emploi à l'international, retour dans la région d'origine, éducation des enfants sont autant de motifs évoqués. Cette priorisation en faveur de la vie privée marque la fin sans retour d'une séquence (plus ou moins longue) dans une carrière jusqu'alors consacrée au journalisme. Par exemple, Adrien interrompt au bout de sept ans sa carrière de journaliste, alors qu'il occupe un poste de rédacteur 
en CDI dans une rédaction parisienne. Il change radicalement de mode de vie après cette « rupture », pour rejoindre Bordeaux et devenir rédacteur en chef dans une collectivité territoriale. Il insiste sur la nouvelle direction donnée à sa vie à la faveur de cette mobilité à la fois géographique et professionnelle :

«En l'occurrence, j'ai privilégié ma vie à mon métier et je suis parti vivre à Bordeaux. [...] J'ai juste revu l'importance que j'accordais à mon travail, en fait. [...] Quand on est jeune diplômé et qu'on sort des études, on ne voit a priori que par son métier en se disant, voilà, il faut qu'on se réalise, qu'on est au début d'une carrière professionnelle et évidemment, oui, j'ai beaucoup relativisé la place même de mon métier et des choix dans ma vie » (Adrien, trente-sept ans, rédacteur en chef dans une collectivité territoriale, formation non reconnue, promotion 2007).

\section{5. "Jeter l'éponge "}

De nombreux récits de parcours insistent sur «l'usure » professionnelle liée à la répétitivité des tâches de rédacteur et à la standardisation des contenus d'actualité. Lucie, diplômée de l'IEP de Lyon et (en 2007) de l'EJCAM, devenue responsable marketing en CDI dans le secteur industriel, raconte ainsi son décrochage progressif du journalisme radio :

«Moi, faire de l'information tous les jours et ne pas avoir de temps, faire trois sujets par jour et traiter à la fois... Alors, il y a eu des sujets qui étaient intéressants puis des sujets dont je me contrefichais absolument, type la foire aux cochons. Et çaj'en avais quand même pas mal. » Elle ajoute, à propos des formats toujours plus courts : « $Y a$ pas le temps aujourd'hui d'une manière générale, que ce soit à la radio ou à la télé, et là je parle de temps vraiment spécifique, le sujet le plus long c'est une minute trente. En une minute trente vous ne pouvez pas dire grand-chose " (Lucie, trente-six ans, responsable marketing en CDI, formation reconnue, promotion 2007). 
Certains « sortants » des mondes de l'information relatent une litanie d'expériences décevantes, jusqu'à un point de non-retour à l'issue duquel ils infléchissent leur carrière. Ce mouvement de bascule vers d'autres métiers s'opère progressivement ou brutalement, mais souvent de façon subie. Lucie se remémore en ces termes les raisons pour lesquelles elle a quitté le journalisme radio :

"Parce qu'à un moment donné, il y a des gens comme moi qui se sont rendus compte sur le terrain que c'est pas forcément ce qu'ils voulaient faire au final. Mais dans la décision, il y a aussi beaucoup le sentiment de bosser pour pas grand-chose et d'être pris aussi comme de la matière qu'on pouvait prendre et jeter à sa guise. Mais ça c'est pas spécifique au journalisme, c'est malheureusement valable pour pas mal de boulots » (Lucie, trente-six ans, responsable marketing en $\mathrm{CDI}$, formation reconnue, promotion 2007).

\subsection{Passer " du côté obscur"}

La grande majorité des « sortants » se tournent vers les métiers de la communication. Ce secteur d'activité est toutefois fortement déprécié parmi les professionnels de l'information, fournissant des figures professionnelles contre-identitaires auxquelles s'opposent les « vrais » journalistes. Véritable transgression - pour certains, il s'agit d'une trahison des valeurs et des idéaux du journalisme - le passage à la communication (ou «à l'ennemi ») revient, selon une célèbre réplique cinématographique reprise par une enquêtée, à passer «du côté obscur de la force ».

Les récits des « sortants » font fréquemment référence à la dégradation du contenu éditorial et des conditions du travail journalistique, mais aussi à une double contrainte politique et économique pesant tout particulièrement sur la presse locale. C'est le cas d'Isabelle, quarante-sept ans, diplômée de Sciences Po et (en 1997) de l'ESJ de Lille, qui devient, après cinq années d'expérience dans la presse régionale du Nord de la France, rédactrice en chef contractuelle des publications éditées par une collectivité locale. Si elle y trouve «un métier plus stable pour [elle] et [sa] famille », elle explique aussi sa reconversion dans la communication territoriale par les motifs suivants : 
«On ne faisait pas de l'écriture mais de la communication. Il faut plaire à machin, ne pas décevoir machin, faire attention à la concurrence... En plus, dans la presse locale les conditions de travail sont de plus en plus difficiles et les journalistes ont de moins en moins le temps de faire leur travail. Quand j'écrivais un article, on me disait parfois: (imitant) "ah non, untel est furieux car tu as écrit tel article, ça fait une publicité à son concurrent”, etc., donc la pression économique se ressentait énormément. \" (Isabelle, quarante-sept ans, rédactrice en chef des publications dans une collectivité locale, contractuelle, formation reconnue, promotion 1997).

\section{Des emplois instables aux carrières déviantes}

Nous ferons ici l'hypothèse que les parcours de sortie des métiers du journalisme sont aussi le moment et la résultante d'une expérience et d'une épreuve morales, au cours desquelles les « sortants » sont conduits à redéfinir leur identité au travail (Dubar, 2004) et le journalisme au gré de leur progression dans des positions divergentes au sein de la profession. En effet, la plupart des enquêtés sont entrés dans les mondes de l'information par attrait, sinon par vocation, et ils conservent après leur sortie une vision positive du journalisme, dont l'un des rôles consiste à « donner la parole à ceux qui ne l'ont pas ». Mais ces mêmes diplômés se trouvent ensuite bloqués dans des emplois instables, où ils subissent la précarité des conditions d'emploi et de rémunération. Cette précarité les pousse dans des situations de pluriactivité où, nous l'avons vu, coexistent fréquemment le journalisme et la communication. Les trajectoires divergentes sont alors le lieu d'une dépossession des attributs qui dénotent l'appartenance au groupe des journalistes, mais aussi d'une transgression des principes par lesquels la profession affirme sa consistance et son indépendance. Les situations de précarité et de pluriactivité se traduisent souvent par la non-attribution ou le non-renouvellement de la carte de presse, faute de déclarer des revenus suffisants dans les métiers du journalisme. A cette dégradation symbolique s'ajoute la transgression des normes, inculquées au cours de la formation et de la socialisation professionnelles, qui prohibent le mélange des genres entre le journalisme et la communication. 
Dès lors, les trajectoires divergentes peuvent être pensées et vécues comme des carrières « déviantes », au cours desquelles les individus intériorisent, par des «transactions » à la fois objectives et subjectives (Dubar, 2004), leur marginalité au sein des mondes du journalisme. Ces carrières sont déviantes à l'aune des dispositifs qui matérialisent et institutionnalisent l'existence des « journalistes professionnels », ainsi que des rhétoriques professionnelles qui soutiennent cette « fiction » et lui confèrent son efficacité (Ruellan, 2007). Relégués dans les « zones grises » du journalisme (Bouron, Devillard, Le Saulnier, Leteinturier, 2017) et, comme tels, confrontés à « l'expérience des conditions sociales en porte à faux »(Boltanski, 1982, p. 32), les diplômés en viennent à élaborer des contre-modèles professionnels pour justifier leur position et leur condition. Ces contre-modèles professionnels peuvent aussi bien soutenir leur persévérance dans les métiers du journalisme, par des formes de rationalisation de la précarité (Balbastre, 2000), que préparer leur conversion, au sens doctrinaire du terme, aux métiers de la communication.

Par exemple, le récit de parcours d'Amandine, trente-quatre ans, est celui d'un passage progressif du journalisme à la communication, qui l'amène à contester le clivage normatif et à établir des continuités pratiques entre l'un et l'autre. Diplômée en 2007 d'une formation publique spécialisée, elle se confronte à une précarité chronique dans les secteurs de la presse et de la radio locales, et se dirige progressivement vers des activités non journalistiques, jusqu'à créer sa propre agence de communication tout en réalisant ponctuellement des piges. Elle décrit sa conversion à la communication moins comme le deuil que comme la continuation, par d'autres moyens, de son "rêve de gosse » pour le journalisme :

«Avec le temps, j'ai arrêté de me dire que je... ne pouvais être que journaliste. En gros, aujourd'hui... comment dire ça? Quand j'ai commencé on nous disait : (imitant) "la communication c'est pas le journalisme, il n'y a pas de passerelles possibles entre, et la communication c'est le mal". Je vous résume ça de manière très caricaturale mais c'était ça en gros. Du coup pour moi il était hors de question de travailler en communication, et puis au final mes expériences journalistiques n'étaient pas forcément épanouissantes. [...] Et c'est comme ça que j'ai commencé à mettre un pied dans la communication, puisqu'on 
m'a proposé un poste, euh, dans la communication. Mais deux fois par mois je partais en reportage terrain. Royal! $Y$ compris à l'étranger, royal! Et donc, c'est comme ça que j'ai commencé à me dire je vais arrêter de me mettre des barrières à moi-même, de... de mettre des étiquettes, ce qui est très français aussi ! Et puis je vais aller là où je pense qu'il y a du sens pour moi, et où je peux être utile aussi. Et aujourd'hui moi je suis très épanouie dans mes multiples activités, même si j'ai pas de carte de presse ni la qualité de journaliste la moitié de mon temps » (Amandine, trente-quatre ans, fondatrice d'une agence de communication et pigiste auto-entrepreneure, formation non reconnue, promotion 2007).

Les récits de parcours manifestent ainsi, de façon récurrente, la propension des « sortants » à établir des continuités entre leur travail de journaliste et celui qu'ils exercent depuis leur sortie. Ce rapprochement se traduit, pour certains, par un discours qui valorise les similitudes s'agissant du contenu et des modalités de leur travail : «Les gens que je vois ont du mal à comprendre comment on passe du journalisme au digital, et moi je leur réponds que le journalisme fait déjà partie de ce monde-là »(Gérard, quarante-six ans, directeur de création en CDI, formation reconnue, promotion 1997). A cet égard, les dénominations choisies par les « sortants » dans la présentation de leur activité actuelle sur LinkedIn sont significatives : depuis le titre de " rédactrice en chef» ou de « journaliste territorial » au sein d'une collectivité locale, jusqu'à celui de « JRI » (journaliste reporter d'images) dans un ministère. Cela va jusqu'à des formes de déni dans les entretiens, à l'instar d'une enquêtée de la promotion de 2012, ex-pigiste pour divers supports de presse et désormais « casteuse » à temps plein pour une société de production audiovisuelle, qui persiste à se définir comme une authentique journaliste. A l'opposé, le rapprochement avec les métiers de la communication est soutenu par une vision désenchantée du journalisme, dont il est difficile de déterminer si elle est la cause ou l'effet de la reconversion opérée. Les « sortants » concernés insistent sur les logiques de marchandisation de l'information, qui dénaturent le contenu et la valeur du travail des journalistes. Cette dénonciation de l'emprise du marketing rédactionnel dans le journalisme contemporain a pour fonction, et pour vertu, de justifier leur conversion à la communication : 
« En parlant à mes collègues de mon départ de la presse locale, je leur disais en rigolant: "tant qu'à faire de la communication, au moins assumer de faire de la communication!" Quand on doit faire trois ou quatre articles dans la journée, il est évident qu'on fait plus de la communication que de l'information à mes yeux. On est prisonniers du dossier de presse qu'on a récupéré car on n'a pas le temps de faire les recherches de nous-mêmes » (Isabelle, quarante-sept ans, rédactrice en chef des publications dans une collectivité locale, contractuelle, formation reconnue, promotion 1997).

\section{Conclusion}

En somme, quelles sont les conditions de possibilité et, pour ainsi dire, de félicité des parcours de sortie des métiers du journalisme ? En premier lieu, les modalités d'emploi et de rémunération en vigueur dans les entreprises de presse organisent des conditions matérielles propices aux défections. L'extension de la précarité, sinon son institutionnalisation, pousse de nombreux candidats à la profession à se tourner vers des secteurs d'activité où ils pourront espérer des conditions d'emploi et de rémunération plus favorables. Quitte à faire le deuil, pour certains, de la « vocation » ou de l'« idée fixe » qui a motivé leur carrière dans le journalisme. Les défections sont d'autant plus probables parmi les journalistes précaires qu'ils sont fréquemment amenés à cumuler des activités journalistiques et non journalistiques, à cause de l'instabilité ou de l'insuffisance des revenus que leur procure leur profession de référence. Or, ces situations de pluriactivité intersectorielle sont aussi des processus de socialisation dans d'autres métiers qui, s'ils se prolongent, peuvent favoriser le franchissement du Rubicon. De nombreux diplômés sont ainsi passés progressivement, sinon insidieusement, du journalisme à la communication, à la faveur de missions répétées dans le second secteur d'activité, jusqu'à y déclarer l'essentiel de leurs revenus et à perdre la carte de presse, ou jusqu'à y trouver des conditions d'emploi et de rémunération plus avantageuses.

Le capital scolaire (en volume et en structure) accumulé par les diplômés de formations en journalisme constitue une ressource décisive dans ces processus de reconversion. Certes, le passage par une formation reconnue par la profession a un effet protecteur, au sens 
où il diminue les probabilités de sortie de la profession (Leteinturier, 2016). Pour autant, un haut niveau de qualification justifie des prétentions statutaires et salariales qui se voient contrariées par l'assignation à des emplois instables. Ainsi, les enquêtés expriment fréquemment le décalage éprouvé entre, d'un côté, les performances scolaires et les aspirations professionnelles qu'elles soutiennent, et, de l'autre, les probabilités très incertaines d'accéder à un emploi stable et rémunérateur dans les métiers du journalisme. Ce sentiment est exacerbé parmi les diplômés ayant passé avec succès toutes les épreuves de l'excellence scolaire (depuis les filières élitistes jusqu'à la "voie royale » des écoles reconnues) mais cantonnés aux marges de la profession et confrontés à une précarité durable. De nombreux enquêtés partagent ainsi la condition des «dominés aux études longues » (Collovald \& Mathieu, 2009, p. 122). Dans ces conditions, ils peuvent être tentés de valoriser leur capital scolaire en se tournant vers des secteurs d'activité où ils pourront en espérer un meilleur rendement. Les reconversions étant facilitées par la valeur sociale attachée, sur le marché de l'emploi en général et sur celui de la communication en particulier, aux diplômes attribués par les formations labellisées.

En troisième lieu, l'analyse des mobilités intersectorielles au sein de l'échantillon suggère que certains secteurs informationnels sont davantage propices aux reconversions professionnelles. Certains diplômés travaillent, au moment de leur sortie, dans la presse spécialisée grand public, en ligne ou hors ligne, ou dans la presse technique et professionnelle. Ils sont ainsi amenés à se spécialiser sur les thématiques couvertes par les supports de presse où ils exercent. Pareille spécialisation, par les compétences et les appétences, mais aussi par le voisinage qu'elle engage, offre des opportunités de reconversion (Chupin \& Mayance, 2013). D'autres diplômés se recrutent dans le pôle le plus commercial du champ journalistique. Ce pôle se caractérise par une forte perméabilité aux logiques du marketing et de la publicité, laquelle peut faciliter, là aussi, les passages du journalisme à la communication (Frisque, 2014). Dans les deux cas, les mobilités professionnelles sont facilitées par la transférabilité, d'un secteur d'activité à l'autre, des ressources accumulées dans les mondes de l'information, à savoir la familiarité avec les logiques de travail des journalistes, les compétences techniques, mais aussi le capital social (le « carnet d'adresses »). Cette transférabilité permet à de nombreux enquêtés d'établir des continuités entre leurs activités passées et présentes. 
Enfin, les récits de parcours font apparaître les opérations subjectives et, plus spécifiquement, les « transactions » identitaires (Dubar, 2004) qui soutiennent les processus de reconversion dans la communication. Au sein des porosités structurelles et des déplacements individuels entre les mondes de l'information et ceux de la communication s'élaborent des contre-modèles professionnels, lesquels brouillent davantage les frontières et l'identité (déjà troubles) du journalisme « professionnel ». En toute hypothèse, les carrières divergentes (Bastin, 2015 ; Basti \& Machut, 2016) peuvent aussi se penser comme des carrières déviantes, au sens où elles engagent une série d'écarts, sinon de transgressions, vis-à-vis de la profession, de ses attributs matériels et symboliques, et, tout autant, de ses standards normatifs. En retour, la matérialisation et, conjointement, l'intériorisation des valeurs attachées à ces occupations déviantes confortent les parcours de sortie du journalisme.

\section{Références}

Accardo, A. (dir.) (1998). Journalistes précaires. Bordeaux : Le Mascaret.

Balbastre, G. (2000). Une information précaire. Actes de la Recherche en Sciences sociales 131/132, 76-85. Disponible à : https://www.cairn.info/revue-actes-de-larecherche-en-sciences-sociales-2000-1-page-76.htm

Bastin, G. (2015). Le Paradoxe du paria. Morphologie des mondes de l'information et persona des journalistes. Paris : EHESS. Mémoire de synthèse pour l'HDR.

Bastin, G. (2016). L'approche morphologique des mondes de l'information : modèles et données pour l'analyse séquentielle de la personnalité des journalistes. Recherches en communication 43, 5-26. Disponible à : https://doi.org/10.14428/rec. v43i43.48743

Bastin, G. \& Machut, A. (2016). Gravitation et dispersion dans les carrières des journalistes passés par la presse quotidienne nationale. Temporalités [En ligne], 23, mis en ligne le 12 octobre 2016. URL : http://temporalites.revues.org/3403

Boltanski, L. (1982). Les Cadres. La formation d'un groupe social. Paris : Les Éditions de Minuit.

Bouron, S., Devillard, V., Le Saulnier, G. \& Leteinturier, C. (2017). L'Insertion et les parcours professionnels des diplômés de formations en journalisme. Paris : CARISM. Rapport pour la CPNEF Audiovisuel, la CPNEF Journaliste, la CPNEF Presse, et l'AFDAS.

Cardon, V. \& Roharik, I. (2016). Le déploiement temporel des inégalités de genre dans le monde du journalisme. Une analyse longitudinale. Temporalités [En ligne], 23, mis en ligne le 10 octobre 2016. URL : http://journals.openedition.org/temporalites $/ 3386$

Chupin, I. \& Mayance, P. (2013). Au service de « La Profession ». Journalistes et communicants pris dans le secteur agricole. Politiques de communication 1(1), 241-268. Disponible à : https://doi.org/10.3917/pdc.001.0241 
Collovald, A. \& Mathieu, L. (2009). Mobilisations improbables et apprentissage d'un répertoire syndical. Politix 86(2), 119-143. Disponible à : https://doi.org/10.3917/ pox.086.0119

Darmon, M. (2008). La notion de carrière : un instrument interactionniste d'objectivation. Politix 82(2), 149-167. Disponible à : https://hal.archives-ouvertes.fr/ halshs-00425847/

Devillard, V. (2002). Les trajectoires des journalistes détenteurs de la carte de presse entre 1990 et 1998. La montée de la précarité. Communication et langages 133, 21-32. Disponible à : https://www.persee.fr/doc/colan_0336-1500_2002_ num_133_1_3152

Devillard, V. \& Rieffel, R. (2001). L'insertion professionnelle des nouveaux journalistes : parcours 1990-1998. Dans V. Devillard, M.-F. Lafosse, C. Leteinturier \& R. Rieffel, Les Journalistes français à l'aube de l'an 2000. Profils et parcours. (pp. 123-158). Paris : Éditions Panthéon-Assas.

Dubar, C. (2004). La Socialisation. Construction des identités sociales et professionnelles. Paris : Armand Colin.

Frisque, C. (2014). Précarisation du journalisme et porosité croissante avec la communication. Les Cahiers du journalisme 26, 94-115. Disponible à : http://www.cahiersdujournalisme.net/pdf/26/06_FRISQUE.pdf

Frisque, C. \& Saitta, E. (2011). Journalistes de la précarité. Formes d'instabilité et modes d'adaptation. Rennes : CRAPE. Rapport pour le DEPS au ministère de la Culture et de la Communication.

Hughes, E. (1996). Le Regard sociologique. Essais choisis. Paris : Éditions de l'EHESS.

Lafarge, G. (2019). Les Diplômés du journalisme. Sociologie générale de destins singuliers. Rennes : Presses universitaires de Rennes.

Lafarge, G. \& Marchetti, D. (2011). Les portes fermées du journalisme. L'espace social des étudiants des formations « reconnues ». Actes de la Recherche en Sciences sociales 189(4), 72-99. Disponible à : https://doi.org/10.3917/arss.189.0072

Lefresne, F. (2003). Les Jeunes et l'emploi. Paris : La Découverte.

Leteinturier, C. (2014). Communication et carrières des journalistes français : le cas des refus de la carte de presse 2010. Les Cahiers du journalisme 26, 116-133. Disponible à : http://www.cahiersdujournalisme.net/pdf/26/07_LETEINTURIER_TAB. pdf

Leteinturier, C. (2016). Continuité/discontinuité des carrières des journalistes français encartés. Étude de deux cohortes de nouveaux titulaires de la carte de presse. Recherches en communication 43, 27-55. Disponible à : https://doi.org/10.14428/ rec.v43i43.48753

Leteinturier, C. \& Frisque, C. (dir.) (2015). Les Espaces professionnels des journalistes. Des corpus quantitatifs aux analyses qualitatives. Paris : Éditions Panthéon-Assas.

Marchetti, D. \& Ruellan, D. (dir.) (2001). Devenir journalistes. Sociologie de l'entrée sur le marché du travail. Paris : La Documentation française.

Ruellan, D. (2007). Le Journalisme ou le professionnalisme du flou. Grenoble : Presses universitaires de Grenoble.

SCAM (2013). De quoi vivent les journalistes ? Enquête SCAM [En ligne]. URL : http://www.scam.fr/Portals/0/Dossiers/DPenquete_journalistesnov2013.pdf?ver=2015-10-29-174312-910 
Standaert, O. (2016a). À l'orée du journalisme, aux marges de ses idéaux. Marchés du travail et trajectoires d'insertion des nouveaux journalistes de Belgique francophone. Questions de communication 30, 335-354. Disponible à : https://doi.org/10.4000/ questionsdecommunication. 10805

Standaert, O. (2016b). La continuité des carrières au régime flexible : décentrement et dispersion à l'orée du marché du journalisme. Recherches en communication 43, 57-77. Disponible à : https://doi.org/10.14428/rec.v43i43.51233

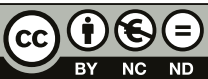

«Attibution - pas d'utilisation Commerciale - Pas de Modification 4.0. International» (CC BY-NC-ND) 